\title{
Cytotoxicity and enhancement activity of essential oil from Zanthoxylum bungeanum Maxim. as a natural transdermal penetration enhancer"
}

\author{
Yi LAN ${ }^{1}$, Qing WU ${ }^{\dagger 1}$, Ying-qiu MAO ${ }^{2}$, Qiong $\mathrm{WANG}^{1}$, Jing $\mathrm{AN}^{1}$, Yan-yan $\mathrm{CHEN}^{1}$, \\ Wen-ping WANG ${ }^{1}$, Bo-chen $\mathrm{ZHAO}^{1}$, Na LIU ${ }^{1}$, Ye-wen ZHANG ${ }^{1}$ \\ ( ${ }^{1}$ School of Chinese Materia Medica, Beijing University of Chinese Medicine, Beijing 100102, China) \\ $\left({ }^{2}\right.$ Center of Science Experiment, Beijing University of Chinese Medicine, Beijing 100029, China) \\ ${ }^{\dagger}$ E-mail: qwu@vip.sina.com \\ Received Aug. 28, 2013; Revision accepted Nov. 13, 2013; Crosschecked Jan. 11, 2014
}

\begin{abstract}
The aim of this present study is to investigate the effect of Zanthoxylum bungeanum oil (essential oil from $Z$. bungeanum Maxim.) on cytotoxicity and the transdermal permeation of 5-fluorouracil and indomethacin. The cytotoxicity of $Z$. bungeanum oil on dermal fibroblasts and epidermal keratinocytes was studied using an MTT (3-(4,5-dimethylthiazol-2-yl)-2,5-diphenyltetrazolium bromide) assay. The rat skin was employed to determine the percutaneous penetration enhancement effect of $Z$. bungeanum oil on hydrophilic and lipophilic model drugs, i.e., 5 -fluorouracil and indomethacin. The secondary structure changes of the rat stratum corneum (SC) were determined using attenuated total reflectance-Fourier transform infrared spectroscopy (ATR-FTIR), and saturated solubilities and $\mathrm{SC} /$ vehicle partition coefficients of two model drugs with and without $Z$. bungeanum oil were also measured to understand its related mechanisms of action. It was found that the half maximal inhibitory concentration $\left({ }^{\prime} C_{50}\right)$ values of $Z$. bungeanum oil were significantly lower in $\mathrm{HaCaT}$ and CCC-ESF-1 cell lines compared to the well-established and standard penetration enhancer Azone. The $Z$. bungeanum oil at various concentrations effectively facilitated the percutaneous penetration of two model drugs across the rat skin. In addition, the mechanisms of permeation enhancement by $Z$. bungeanum oil could be explained with saturated solubility, SC/vehicle partition coefficient, and secondary structure changes of SC.
\end{abstract}

Key words: Zanthoxylum bungeanum Maxim., Essential oil, Transdermal delivery, Penetration enhancer, HaCaT, Attenuated total reflectance-Fourier transform infrared spectroscopy (ATR-FTIR)

\section{Introduction}

The pericarp of Zanthoxylum bungeanum Maxim., belonging to the Rutaceae family, is commonly used as a flavoring and traditional Chinese medicine for its

\footnotetext{
ॠCorresponding author

* Project supported by the National Natural Science Foundation of China (No. 81073059), the Beijing Natural Science Foundation (No. 7132127), and the Innovative Research Team in Beijing University of Chinese Medicine (No. 2011-CXTD-13), China

(c) Zhejiang University and Springer-Verlag Berlin Heidelberg 2014
}

flavors and medicative characteristics in China. According to the theory of traditional Chinese medicine (TCM), the pericarp of $Z$. bungeanum Maxim. possesses the medical functions of warming the spleen and stomach to relieve pain and killing parasites to relieve itching, and it is also effective for the therapy of pathogenic wind, epigastric pain, eczema, pruritus, fungal infection, diarrhea, and dysentery (Gong et al., 2009; Wei et al., 2011; Zhu et al., 2011). In addition, $Z$. bungeanum oil is also widely used for topical remedies. According to the statement on Li Yue Pian 
Wen (published in 1870), an ancient classic literature on topical remedies in China, these herbs like $Z$. bungeanum Maxim., peppermint oil, which are rich in essential oil, can effectively promote the percutaneous absorption of the active components in a prescription for their unique properties.

In our previous studies, Zhitong cataplasm, a clinical empirical formula mainly consisting of the extracts of four herbs, Pericarpium Zanthoxyli ( $Z$. bungeanum Maxim.), Rhizoma Chuanxiong, Radix Angelicae Dahuricae, and Herba Asari, was developed and topically used for the treatment of rheumatoid arthritis and joint pain. We found that $Z$. bungeanum oil in this prescription had the function of facilitating the percutaneous absorption of the active components besides its medicative function as described above. However, there is currently no systematic information available about the skin permeation enhancement effect of $Z$. bungeanum oil. It is well known that it is a prerequisite to enable the drug molecule to pass through the skin for the exertion of drug pharmacological activity. Furthermore, most of the essential oil is mainly composed of terpenes (Gong et al., 2009; Xia et al., 2011), which have been generally reported to enable the drug molecule to penetrate across the skin as a penetration enhancer (Aqil et al., 2007; Sapra et al., 2008). The Z. bungeanum oil is thus supposed to possess the properties of facilitating the percutaneous absorption of the components in a prescription besides its medicative characteristics. In addition, the $Z$. bungeanum oil appears to offer low toxicity potential for use in transdermal formulations due to its natural origin (Fox et al., 2011).

Hence, the aim of this study is to investigate the toxicity and percutaneous penetration enhancement activity of $Z$. bungeanum oil and its underlying mechanisms. Considering the diverse properties of the components penetrating through the skin, 5-fluorouracil (5-FU, $n$-octanol/water partition coefficient $\log K_{\mathrm{o} / \mathrm{w}}=$ $-0.95)$ and indomethacin (IM, $\left.\log K_{\mathrm{o} / \mathrm{w}}=3.80\right)$ were chosen as hydrophilic and lipophilic model drugs, respectively (Zhao et al., 2008). In addition, rat skin was employed to monitor the skin permeation enhancement effect of $Z$. bungeanum oil on the transdermal delivery of model drugs.

\section{Materials and methods}

\subsection{Drugs and chemicals}

5-FU and IM were obtained from Beijing Ouhe Technology Co., Ltd. and Suzhou Yacoo Co., Ltd., China, respectively. 1,8-Cineole, limonene, and terpinen-4-ol were supplied by the TCI Tokyo Chemical Industry Co., Ltd., Japan. Propylene glycol (PG) was purchased from Beijing Chemical Reagent Co., Ltd., China. Dimethyl sulfoxide (DMSO), 3-(4,5-dimethylthiazol-2-yl)-2,5-diphenyltetrazolium bromide (MTT), and $\mathrm{C}_{8}-\mathrm{C}_{20} n$-alkanes were obtained from Sigma-Aldrich (Shanghai, China). Azone was purchased from Beijing Changhua Fine Chemical Co., Ltd., China. Acetonitrile and methanol of highperformance liquid chromatography (HPLC) grade were supplied by Merck KGaA (Germany). All other chemicals used were of analytical grade.

\subsection{Plant materials and essential oil extraction}

The dried fruits of $Z$. bungeanum used in this work were purchased from Anguo Lulutong Co., Ltd. (Hebei, China), and identified by Prof. Shou-ying DU (Department of Chinese Pharmacy, Beijing University of Chinese Medicine, China). These specimens were harvested in August, 2012. A voucher specimen was deposited in the Department of Chinese Pharmacy, Beijing University of Chinese Medicine, China. The dried herbs $(2.0 \mathrm{~kg})$ were subjected to hydrodistillation in 5 volumes of water for $3 \mathrm{~h}$ by using a Clevenger-type apparatus. The yellowish essential oil was obtained in a yield of $2.9 \%(\mathrm{w} / \mathrm{w})$ after drying with anhydrous sodium sulphate and stored under $\mathrm{N}_{2}$ at $4{ }^{\circ} \mathrm{C}$ in a sealed brown vial until tested and analyzed.

\subsection{Cell line and culture}

HaCaT (epidermal keratinocytes) and CCCESF-1 (dermal fibroblasts) cell lines were obtained from the Cell Resource Center of Institute of Basic Medical Sciences, Chinese Academy of Medical Sciences (Peking Union Medical College, China). The cells were incubated in Minimum Essential Medium (MEM Eagles with Earle's Balanced Salts) and Dulbecco's Modified Eagle's Medium (DME H-21 $4.5 \mathrm{~g} / \mathrm{L}$ glucose), respectively, supplemented with 
$10 \%$ fetal bovine serum (FBS), $100 \mathrm{U} / \mathrm{ml}$ penicillin/ streptomycin at $37{ }^{\circ} \mathrm{C}$ in a $5 \% \mathrm{CO}_{2}$ incubator. $\mathrm{HaCaT}$ cells were fusiform under a microscope (Fig. 1c), and CCC-ESF-1 cells were fibroblast-like cells (Fig. 1d).

\subsection{HPLC methodology of model drugs}

The HPLC system for analyzing drug concentrations was equipped with an SPD-20A variablewavelength ultraviolet absorbance detector, two LC-20AT pumps, and computer integrating system (Shimadzu, Kyoto, Japan). Alltima AQ ODS column $(250 \mathrm{~mm} \times 4.6 \mathrm{~mm}$ i.d., $5 \mu \mathrm{m}$ particle size; Grace) and Purospher STAR RP-18 column $(250 \mathrm{~mm} \times 4.6 \mathrm{~mm}$ i.d., $5 \mu \mathrm{m}$ particle size; Merck) were used to determine the contents of 5-FU and IM, respectively. For 5-FU, the mobile phase was distilled water at a flow rate of $1 \mathrm{ml} / \mathrm{min}$. 5-FU was detected at $266 \mathrm{~nm}$ with the retention time of $7.5 \mathrm{~min}$. For IM, the mobile phase was a mixture of acetonitrile $/ 0.1 \mathrm{~mol} / \mathrm{L}$ acetic acid $(45: 55, \mathrm{v} / \mathrm{v})$ at the flow of $1 \mathrm{ml} / \mathrm{min}$. IM was detected at $231 \mathrm{~nm}$ with the retention time of $12.5 \mathrm{~min}$ Calibration curves for 5-FU and IM were linear over the ranges of $0.5-500 \mu \mathrm{g} / \mathrm{ml}$ and $0.5-250 \mu \mathrm{g} / \mathrm{ml}$, respectively. The coefficients of variation for intra- and inter-day variations were below $1 \%$.

\subsection{Gas chromatography-mass spectral (GC-MS) analysis}

The $Z$. bungeanum oil was subjected to GC-MS analysis on a Shimadzu system (Shimadzu QP-2010, Japan) equipped with a National Institute of Standards and Technology (NIST) database and a DB-1MS capillary column $(30 \mathrm{~m} \times 0.25 \mathrm{~mm} \times 0.25 \mathrm{~mm}$, Agilent). Helium $(99.99 \%)$ was the carrier gas at a flow rate of $1.0 \mathrm{ml} / \mathrm{min}$. The oven temperature was held at $50{ }^{\circ} \mathrm{C}$ for $20 \mathrm{~min}$ and then programmed from 50 to $200^{\circ} \mathrm{C}$ at a rate of $2{ }^{\circ} \mathrm{C} / \mathrm{min}$. The injector temperature, MS transfer line and ion source temperatures were set at 250, 280, and $250{ }^{\circ} \mathrm{C}$, respectively. Mass scanning was in the range of $45-500 \mathrm{amu}$. The split ratio was 1:20. An aliquot $(1.0 \mu \mathrm{l})$ of the diluted sample $(1 / 100$ in $n$-hexane, w/v) was injected automatically.

The constituents of the essential oil were identified by matching their MS fragmentation patterns with those in NIST 2008 mass spectral library and also by comparing their Kovat retention indices, which were determined by injection of the sample with a solution containing the homologous series of $\mathrm{C}_{8}-\mathrm{C}_{20}$ $n$-alkanes as reported before (Sereshti et al., 2011; Wu et al., 2012). Moreover, the major oil contributors were further confirmed by comparing the corresponding pure standard compounds. The percentage composition was computed by the normalization method from the GC peak areas.

\subsection{Determination of physicochemical properties of essential oil}

For recognition of the basic physiochemical properties of $Z$. bungeanum oil, an ultraviolet-visible spectroscopy (UV-VIS) spectrophotometer (SP-752, Shanghai Spectrum Instruments Co., Ltd., China) was employed to monitor its maximum UV absorption wavelength. An automatic surface tensiometer (JK99B, Shanghai Zhongcheng Digital Technology Instruments Co., Ltd., China) was adopted to detect the surface tension of the essential oil. The conductivity of the oil was determined using a DDS-307 conductivity meter (Shanghai Jingke Electronic Co., Ltd., China). The $\mathrm{pH}$ value measurement was made using a pH meter (PHS-3C, Sanxin, China) at $25^{\circ} \mathrm{C}$. The relative density was measured through a weighing method using an electronic balance (Sartorius, Germany).

\subsection{Preparation of full thickness skin and stratum corneum (SC)}

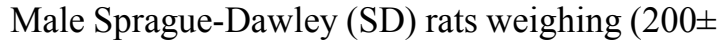
10) $g$ were purchased from Shibeifu Laboratory Animal Technology Co., Ltd. (Beijing, China). After sacrificing the rats with excess ether inhalation, hair from the abdominal surface was removed with an animal hair clipper (Codos, China) with an extreme precaution as to not impair the skin. The shaved skin was then excised from the animals, the subcutaneous tissue was removed surgically and the dermal side was wiped with a cotton swab to remove the adhered fat tissue. The full thickness skin prepared was subsequently washed with phosphate buffer saline (PBS), wrapped in aluminum foil, and stored at $-20^{\circ} \mathrm{C}$ (used within two weeks). All the animal experiments were conducted in compliance with the Guiding Principles for the Care and Use of Laboratory Animals, Beijing University of Chinese Medicine, China, and the experimental protocol was approved by the Committee on Animal Research of the Beijing University of 
Chinese Medicine.

The excised rat skin was treated with $2 \mathrm{~mol} / \mathrm{L}$ sodium bromide solution for $8 \mathrm{~h}$ as reported previously (Jain et al., 2002). The SC samples were separated by using a cotton swab moistened with double distilled water. SC sheets were thoroughly washed with water and dried in a vacuum desiccator.

\subsection{Cytotoxicity assay}

To determine the cellular toxicity of $Z$. bungeanum oil in epidermal keratinocyte and dermal fibroblast cultures, the MTT assay was used to monitor the toxic effects of $Z$. bungeanum oil on skin cells in vitro as described in detail elsewhere (Song et al., 2005). Fibroblasts or keratinocytes were seeded into 96-well plates at a density of 7000 cells in $100 \mu 1$ medium per well. After $24 \mathrm{~h}$, the cells were incubated with varying concentrations of enhancer solution in a culture medium with $1 \%$ DMSO (as a vehicle) for $24 \mathrm{~h}$ at $37^{\circ} \mathrm{C}$. The cells that were treated with culture medium containing 1\% DMSO were used as the control. Subsequently, the medium was removed and supplemented with fresh medium containing $20 \mu \mathrm{l}$ MTT solution $(5 \mathrm{mg} / \mathrm{ml}$ in phosphate buffer) and the cells were incubated for $4 \mathrm{~h}$. Then the medium was replaced with $150 \mu \mathrm{L}$ DMSO to dissolve the formazan crystals. The plate was incubated for 10 min while shaking. Absorbance was read at $490 \mathrm{~nm}$ using a microplate spectrophotometer (Thermo Scientific, Finland). The half maximal inhibitory concentrations $\left(\mathrm{IC}_{50}\right)$ were calculated using SPSS software v16.0 according to the previously described method (Xiang et al., 2010).

\subsection{Skin permeation studies}

The vertical Franz-type diffusion cell (Shanghai Kaikai Technology Trade Co., Ltd., China) with a diffusional area of $1.77 \mathrm{~cm}^{2}$ and a receptor chamber volume of $7 \mathrm{ml}$ was employed to investigate percutaneous penetration enhancement activity of the $Z$. bungeanum oil. The epidermis prepared was sandwiched between the diffusion cells with the SC side up and the dermal side exposed to the receiver compartment containing isotonic PBS $(\mathrm{pH} 7.2)$, the receptor cells were thermostated at $32{ }^{\circ} \mathrm{C}$, and the solution in the receptor chambers was stirred continuously at $300 \mathrm{r} / \mathrm{min}$. The skin was then treated with
$2 \mathrm{ml}$ of varying concentrations of $Z$. bungeanum oil in PG:water $(80: 20, v / v)$. Saturated suspensions of model drugs were used to insure maximum thermodynamic activity and maintain sink conditions. The control was treated with a vehicle only. Samples $(1 \mathrm{ml})$ were withdrawn from the receptor chamber at predetermined time (1, 2, 4, 6, 8, 10, 12, 14, 22, and $24 \mathrm{~h})$, and replaced with an equivalent volume of buffer solution, and the samples were immediately assayed using the HPLC method described in Section 2.4.

\subsection{Determination of saturated solubility of model drugs}

In order to investigate the effect of $Z$. bungeanum oil applied on the saturation solubility of the model drugs, an excess drug was added to the known volumes of the vehicle with or without varying concentrations of $Z$. bungeanum oil, vortexed for $3 \mathrm{~min}$ followed by sonication for $15 \mathrm{~min}$ to dissolve the drug, and then equilibrated at $(32 \pm 0.5){ }^{\circ} \mathrm{C}$ for more than $48 \mathrm{~h}$. The saturated solution was then centrifuged at $10000 \mathrm{r} / \mathrm{min}$ for $15 \mathrm{~min}$ and aliquots of supernatant were filtered through a $0.45-\mu \mathrm{m}$ nylon filter and diluted by a mobile phase before HPLC analysis.

\subsection{Measurement of SC/vehicle partition coeffi- cient of model drugs}

The partition coefficient of model drugs into SC with or without various concentrations of $Z$. bungeanum oil treatment was measured as reported previously (Song et al., 2005). The SC samples were pulverized in a mortar with a pestle. One milliliter of varying concentrations of $Z$. bungeanum oil in PG:water $(80: 20, \mathrm{v} / \mathrm{v})$ containing $0.1 \mathrm{~g} / \mathrm{ml}$ model drug was added to $10 \mathrm{mg}$ ground SC with frequent vortexing. The control was treated with a vehicle only. The mixture was equilibrated for $10 \mathrm{~h}$ at $37^{\circ} \mathrm{C}$. The supernatant solution was obtained by centrifuging at $10000 \mathrm{r} / \mathrm{min}$ for $10 \mathrm{~min}$ and then analyzed for the drug content. The amount of drug bound to the SC was calculated by subtracting the amount of the drug in the supernatant from the initial drug concentration. All partition studies were conducted in triplicate. The partition coefficient $(K)$ of the model drug was obtained using the following equation: $K=$ (drug concentration in $\mathrm{SC}) /($ drug concentration in vehicle). 


\subsection{Fourier transform infrared spectroscopy (FTIR) studies}

The dried SC sheet was cut into approximately $1 \mathrm{~cm}^{2}$ pieces and incubated for $12 \mathrm{~h}$ in $5 \mathrm{ml}$ of the respective solutions at room temperature, where the solutions were $1 \%, 3 \%, 5 \%$, and $10 \% \mathrm{Z}$. bungeanum oil, $0.2 \mathrm{~mol} / \mathrm{L}$ Azone, and solvent (PG:water=80:20 $(\mathrm{v} / \mathrm{v})$, used as the control), respectively. The SC was then cleaned carefully with distilled water to remove the residual solvent on the SC surface and placed in a vacuum desiccator at $37{ }^{\circ} \mathrm{C}$ overnight for complete dehydration. The spectral measurements of all pieces were made with a Nexus FTIR spectrometer (Thermo Nicolet, USA) equipped with an attenuated total reflectance (ATR) attachment with the following parameters: resolution of $2 \mathrm{~cm}^{-1}$, scanning times of 100 , and scanning range of $650-4000 \mathrm{~cm}^{-1}$. The FTIR spectral curves in each group were recorded using an OMNIC 6.2 program attached to the IR instrument itself.

\subsection{Data and statistical analysis}

The parameters for the skin permeation studies were calculated by plotting the cumulative amount of drug permeated across the skin against time (h). Steady state flux $\left(J_{\mathrm{s}}\right)$ was calculated as the slope of the linear portion of the plot (between 8 and $14 \mathrm{~h}$ ). The lag time $\left(T_{\mathrm{lag}}\right)$ was determined by extrapolating the linear portion of the curve to the $X$-axis. The cumulative drug amount in the receptor chamber after $24 \mathrm{~h}$ $\left(Q_{24}\right)$ and diffusion parameter $\left(D / h^{2}\right)$ were calculated from the following equations:

$$
\begin{gathered}
Q_{24}=V_{\mathrm{r}} C_{t}+\sum_{i=0}^{t-1} V_{\mathrm{s}} C_{i}, \\
D / h^{2}=1 /\left(6 T_{\mathrm{lag}}\right),
\end{gathered}
$$

where $C_{i}$ is the drug concentration of the receiver solution at each sampling time, $C_{t}$ is the drug concentration of the sample, $V_{\mathrm{s}}$ and $V_{\mathrm{r}}$ are the volumes of the sampling solution and the receiver solution, respectively, $D$ is the diffusion coefficient within the skin, and $h$ is the diffusional path length.

In order to compare the permeation enhancement capacity of different concentrations of $Z$. bungeanum oil, the enhancement ratio (ER) for flux was calculated using the following equation: $E R=($ flux for skin treated with essential oil)/(flux for control (exposed to only vehicle)).

Data were expressed as the mean \pm standard deviation (SD) and the number of replicates $(n)$ was given in the pertinent figures. A two-tailed Student's $t$-test was used when comparing two different conditions. In all cases, $P<0.05$ was considered significant.

\section{Results and discussion}

\subsection{Chemical compositions and physicochemical properties of the $Z$. bungeanum oil}

A total of 48 compounds were identified from the essential oil, which represented $96.35 \%$ of the total oil. There were 31 constituents accounting for above $0.5 \%$ of the total oil as listed in Table 1, and three major oil contributors (i.e., terpinen-4-ol, 1,8cineole, and limonene, which represented $41.38 \%$ of the total oil) were further confirmed using the corresponding pure compounds. Based on the results of the GC-MS analysis, the $Z$. bungeanum oil contained high contents of oxygenated monoterpenes and monoterpene hydrocarbons, and the major compounds were terpinen-4-ol $(18.42 \%), 1,8$-cineole (15.49\%), limonene (7.47\%), $\alpha$-terpineol (5.79\%), and $\gamma$-terpinene $(5.62 \%)$, which was slightly different from the previous report (Wang et al., 2006) on account of different producing areas, growth conditions, and extracted methods. The results of the measurement of the essential oil physicochemical properties exhibited that the oil had the physiochemical parameters as follows: density $0.86 \mathrm{mg} / \mathrm{ml}$; $\mathrm{pH} 5.18$; electric conductivity $0 \mu \mathrm{s} / \mathrm{cm}$; surface tension $24.96 \mathrm{mN} / \mathrm{m}$; maximum UV absorption wavelength $227 \mathrm{~nm}$.

Generally, the major components determine the biological properties of the essential oil, and it has been reported that some terpenes identified from the essential oil could enhance the transdermal permeation of the polar and non-polar drugs, such as terpinen-4-ol (Magnusson et al., 1997), 1,8-cineole, terpineol (Narishetty and Panchagnula, 2005), and limonene (Okabe et al., 1989), indicating these major oil contributors play a crucial role in the enhancement activity of $Z$. bungeanum oil as a transdermal penetration enhancer. 
Table 1 Major components of the essential oil from Zanthoxylum bungeanum detected by GC-MS

\begin{tabular}{|c|c|c|c|c|}
\hline $\begin{array}{c}t_{\mathrm{R}}^{\mathrm{a}} \\
(\mathrm{min})\end{array}$ & Compound & $\mathrm{RI}^{\mathrm{b}}$ & $\begin{array}{l}\text { ID } \\
\text { method }\end{array}$ & $\begin{array}{c}\text { Relative } \\
\text { content } \\
(\%)\end{array}$ \\
\hline 9.48 & 3-Thujene & 917 & MS, RI & 0.59 \\
\hline 9.81 & $\alpha$-Pinene & 923 & MS, RI & 1.21 \\
\hline 11.99 & $\beta$-Phellandrene & 957 & MS, RI & 1.80 \\
\hline 13.46 & Myrcene & 979 & MS, RI & 3.89 \\
\hline 15.17 & $\alpha$-Terpine & 1004 & MS, RI & 3.29 \\
\hline 15.39 & $o$-Cymene & 1007 & MS, RI & 1.53 \\
\hline 16.02 & 1,8-Cineole & 1013 & $\mathrm{MS}, \mathrm{RI}^{\mathrm{c}}$ & 15.49 \\
\hline 16.28 & Limonene & 1016 & $\mathrm{MS}, \mathrm{RI}^{\mathrm{c}}$ & 7.47 \\
\hline 17.05 & (E)- $\beta$-ocimene & 1024 & MS, RI & 1.19 \\
\hline 17.94 & (Z)- $\beta$-ocimene & 1034 & MS, RI & 0.53 \\
\hline 18.72 & $\gamma$-Terpinene & 1042 & MS, RI & 5.62 \\
\hline 21.36 & Terpinolene & 1071 & MS, RI & 1.69 \\
\hline 22.42 & Linalool & 1082 & MS, RI & 4.55 \\
\hline 23.93 & 1 & 1098 & MS, RI & 1.10 \\
\hline 25.67 & 2 & 1114 & MS, RI & 1.03 \\
\hline 28.69 & Cryptone & 1141 & MS, RI & 1.72 \\
\hline 30.08 & Terpinen-4-ol & 1153 & $\mathrm{MS}, \mathrm{RI}^{\mathrm{c}}$ & 18.42 \\
\hline 31.25 & $\alpha$-Terpineol & 1163 & MS, RI & 5.79 \\
\hline 31.93 & 3 & 1169 & MS, RI & 0.66 \\
\hline 33.20 & 4 & 1180 & MS, RI & 0.58 \\
\hline 34.16 & 5 & 1189 & MS, RI & 0.52 \\
\hline 35.14 & Cumic aldehyde & 1198 & MS, RI & 0.50 \\
\hline 36.91 & Piperitone & 1212 & MS, RI & 2.62 \\
\hline 39.25 & Phellandral & 1232 & MS, RI & 1.08 \\
\hline 40.02 & Linalyl acetate & 1238 & MS, RI & 0.55 \\
\hline 41.91 & Cuminic alcohol & 1254 & MS, RI & 0.52 \\
\hline 50.42 & Terpinyl acetate & 1324 & MS, RI & 4.62 \\
\hline 51.60 & Citronellyl acetate & 1334 & MS, RI & 0.62 \\
\hline 54.75 & Geranyl acetate & 1360 & MS, RI & 0.68 \\
\hline 75.73 & Caryophyllene oxide & 1538 & MS, RI & 0.57 \\
\hline 107.84 & Octadecane & 1843 & MS, RI & 1.32 \\
\hline \multicolumn{3}{|c|}{ Total } & & 91.75 \\
\hline \multicolumn{5}{|c|}{$\begin{array}{l}\text { Compound } 1 \text { : } \text { trans-4-(isopropyl)-1-methylcyclohex-2-en-1-ol; } \\
\text { Compound } \mathbf{2 :} \text { cis-4-(isopropyl)-1-methylcyclohex-2-en-1-ol; } \\
\text { Compound } 3: \text { trans-6-(isopropyl)-3-methylcyclohex-2-en-1-ol; } \\
\text { Compound 4: cis-6-(isopropyl)-3-methylcyclohex-2-en-1-ol; } \\
\text { Compound 5: (Z)-2-methyl-5-(1-methylethenyl)-cyclohexen-2-ol. } \\
\text { a } t_{\mathrm{R}} \text { is the retention time of the identified compounds; }{ }^{\mathrm{b}} \mathrm{RI} \text { is Kovat } \\
\text { retention indices on DB-1MS column; }{ }^{\mathrm{C}} \text { It is further confirmed by } \\
\text { using the corresponding pure compound. ID: identification }\end{array}$} \\
\hline
\end{tabular}

\subsection{Cytotoxicity of $Z$. bungeanum oil on skin cells}

Most penetration enhancers have been shown to produce skin irritation or toxicity in spite of their fairly satisfactory performance in enhancing the permeation of drug molecules; hence few of them have been approved for clinical use. The cytotoxicities of $Z$. bungeanum oil and the well-established and standard penetration enhancer Azone were studied using MTT assay, and the results of keratinocytes and fibroblasts treated with different concentrations of selected penetration enhancers are presented in Fig. 1. All examined permeation enhancers induced dosedependent reductions in cellular viability after $24 \mathrm{~h}$ (Figs. 1a and 1b). As summarized in Table 2, the $\mathrm{IC}_{50}$ values (i.e., the concentration of drugs inducing a $50 \%$ decrease in cell viability) of $Z$. bungeanum oil were prominently higher in both $\mathrm{HaCaT}$ cells and CCC-ESF-1 cells compared to those of Azone, indicating that $Z$. bungeanum oil was probably a low toxic penetration enhancer.

Table $2 \quad \mathrm{IC}_{50}$ values of Azone and the Zanthoxylum bungeanum oil for HaCaT and CCC-ESF-1 cell lines

\begin{tabular}{ccc}
\hline \multirow{2}{*}{$\begin{array}{c}\text { Penetration } \\
\text { enhancer }\end{array}$} & \multicolumn{2}{c}{$\mathrm{IC}_{50}(\mathrm{mg} / \mathrm{ml})$} \\
\cline { 2 - 3 } & $\mathrm{HaCaT}$ & $\mathrm{CCC}-\mathrm{ESF}-1$ \\
\hline Azone & $0.047 \pm 0.002$ & $0.048 \pm 0.004$ \\
Z. bungeanum oil & $2.435 \pm 0.019$ & $3.649 \pm 0.055$ \\
\hline Each value represents the mean $\pm \mathrm{SD}(n=6)$
\end{tabular}

\subsection{Effects of $Z$. bungeanum oil on percutaneous permeation of model drugs}

To determine the permeation enhancement activity of $Z$. bungeanum oil across the rat skin, the control was prepared only by dissolving the model drugs in the base solvent, i.e., PG:water (80:20, v/v). PG:water was chosen as a base solvent on account of its ability to solubilize most tested components, including Z. bungeanum oil and Azone. Moreover, as the well-established and standard penetration enhancer, Azone had been employed by many researchers to achieve significant permeation enhancement and the concentration $(0.2 \mathrm{~mol} / \mathrm{L})$ was typically used in skin penetration enhancement studies (Meidan et al., 2003; Kaushik et al., 2010; Batheja et al., 2011); hence $0.2 \mathrm{~mol} / \mathrm{L}$ Azone was used to compare and better evaluate the permeation enhancement activity of the oil.

The effect of $Z$. bungeanum oil at various concentrations $(1 \%, 3 \%, 5 \%$, and $10 \%)$ on the permeation parameters (flux, $T_{\mathrm{lag}}$, and $Q_{24}$ ) for 5-FU is presented in Table 3 . The oil at higher concentration was not considered in this study on account of its incomplete dissolution in the vehicle. The oil at different 


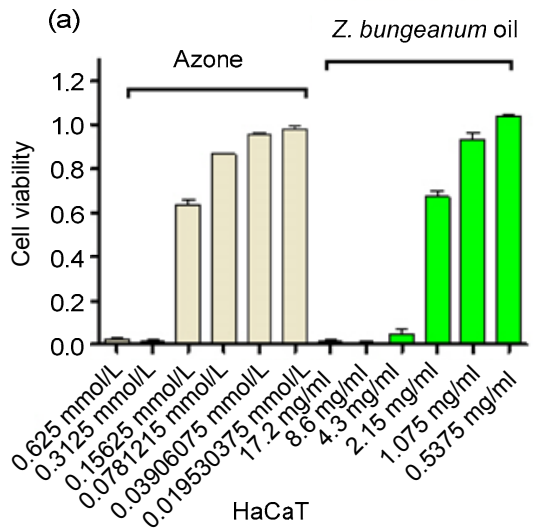

(c)

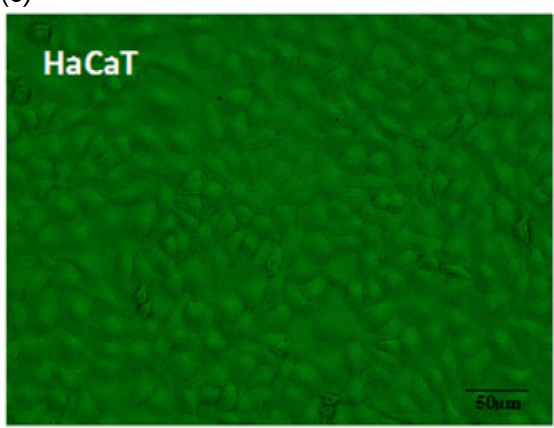

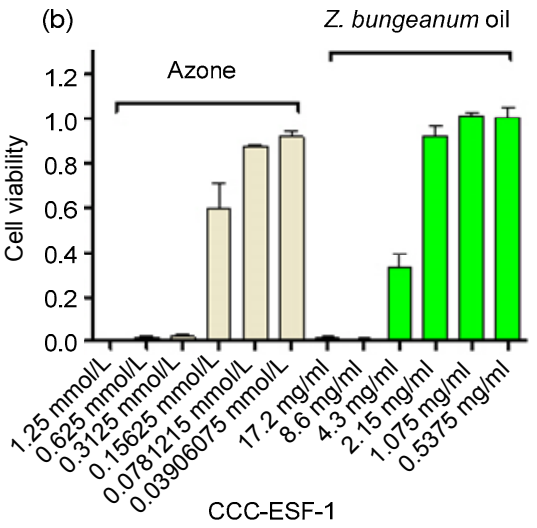

(d)

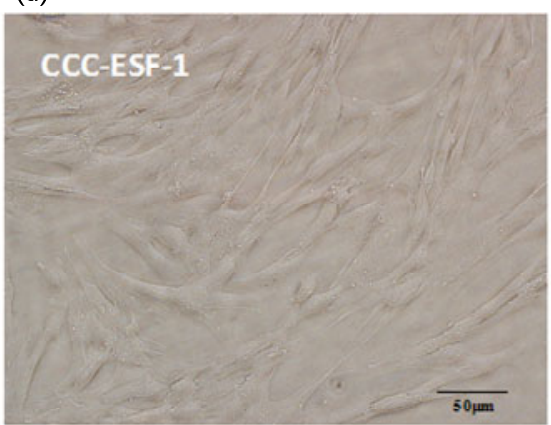

Fig. 1 Toxicities of Zanthoxylum bungeanum oil and Azone in HaCaT keratinocyte and CCC-ESF-1 fibroblast cell lines (a) Effects of selected permeation enhancers on $\mathrm{HaCaT}$ cell viability; (b) Effects of selected permeation enhancers on CCC-ESF-1 cell viability; (c) Cellular morphology of HaCaT; (d) Cellular morphology of CCC-ESF-1. Data in (a) and (b) are expressed as mean $\pm \mathrm{SD}(n=6)$

Table 3 Percutaneous permeation parameters of 5-FU through excised rat skin

\begin{tabular}{ccccrc}
\hline Penetration enhancer & Flux $\left(\mu \mathrm{g} /\left(\mathrm{cm}^{2} \cdot \mathrm{h}\right)\right)$ & $T_{\text {lag }}(\mathrm{h})$ & $Q_{24}\left(\mu \mathrm{g} / \mathrm{cm}^{2}\right)$ & ER & Solubility $(\mathrm{mg} / \mathrm{ml})$ \\
\hline Control & $1.16 \pm 0.21$ & $2.04 \pm 0.83$ & $49.77 \pm 8.85$ & 1.0 & $4.93 \pm 0.40$ \\
0.2 mol/L Azone & $217.96 \pm 20.62^{*}$ & $0.87 \pm 0.22^{*}$ & $7717.26 \pm 701.71^{*}$ & 187.8 & $7.60 \pm 0.46^{*}$ \\
1\% oil & $7.45 \pm 1.19^{*}$ & $5.82 \pm 0.34^{*}$ & $323.24 \pm 51.78^{*}$ & 6.4 & $7.07 \pm 1.88^{*}$ \\
$3 \%$ oil & $102.83 \pm 11.75^{*}$ & $6.48 \pm 0.83^{*}$ & $4949.27 \pm 377.64^{*}$ & 88.6 & $6.72 \pm 0.71^{*}$ \\
$5 \%$ oil & $274.98 \pm 13.13^{*}$ & $5.61 \pm 0.79^{*}$ & $9450.17 \pm 443.77^{*}$ & 237.1 & $3.87 \pm 0.33^{*}$ \\
$10 \%$ oil & $362.06 \pm 43.68^{*}$ & $2.85 \pm 0.69$ & $11180.47 \pm 889.73^{*}$ & 312.1 & $3.58 \pm 0.54^{*}$ \\
\hline
\end{tabular}

Each value represents the mean $\pm \mathrm{SD}(n=5)$, except for ER. ${ }^{*} P<0.05$, statistically significant difference between enhancers and control

concentrations exhibited statistically higher flux and $Q_{24}$ values in comparison to those of the control, and showed a concentration-dependent manner as shown in Fig. 2. Meanwhile, apart from the $10 \%$ oil group, the $T_{\text {lag }}$ value of the other oil groups was significantly superior to that of the control $(P<0.05)$, and there was no significant difference between the control and the $10 \%$ oil group in terms of $T_{\text {lag }}$ value. In addition, $0.2 \mathrm{~mol} / \mathrm{L}$ Azone produced high permeation enhancement activity $(\mathrm{ER}=187.89)$ with low $T_{\text {lag }}$ value compared with the control, which was consistent with the previous studies (Singh et al., 1993; He et al., 2008). However, the $5 \%$ oil group (ER= 237.05) exhibited better penetration enhancement activity than Azone, suggesting that $Z$. bungeanum oil at a proper concentration could effectively promote the transdermal delivery of the hydrophilic drugs with a long $T_{\text {lag. }}$. 


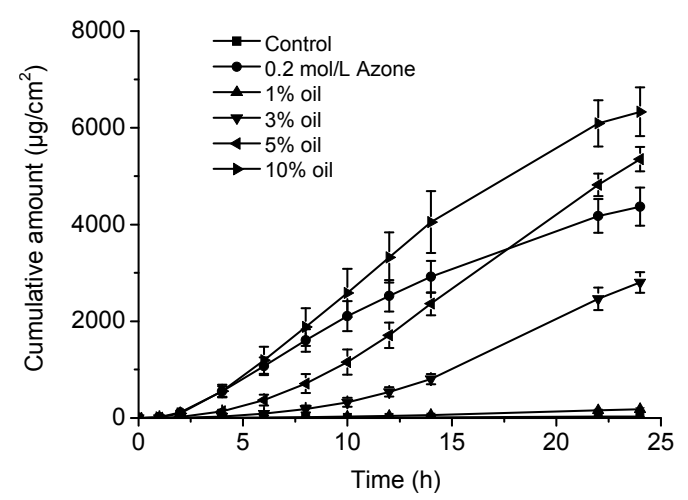

Fig. 2 Effects of Zanthoxylum bungeanum oil on percutaneous permeation of 5-FU through excised rat skin Data are expressed as mean $\pm \mathrm{SD}(n=5)$

The permeation profile of the cumulative amounts of IM penetrated across the rat skin is shown in Fig. 3. Similar to 5-FU, Z. bungeanum oil also markedly enhanced the percutaneous absorption of IM, whereas the $T_{\text {lag }}$ value gradually decreased with increasing the oil concentration as presented in Table 4, implying that $Z$. bungeanum oil remarkably enhanced the penetration of the lipophilic drugs with a relative short $T_{\text {lag. }}$.

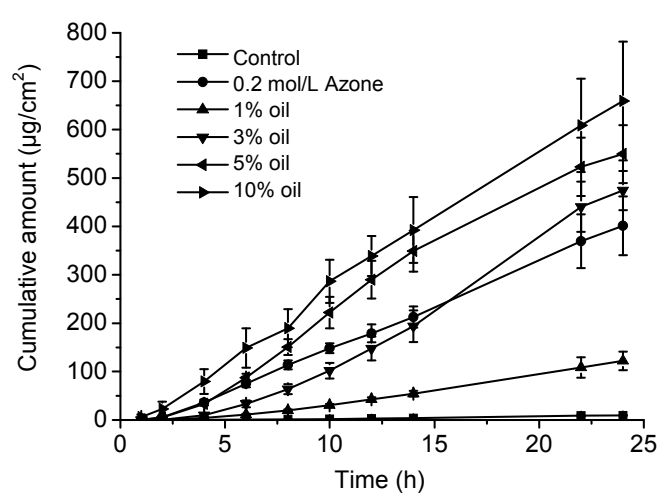

Fig. 3 Effects of Zanthoxylum bungeanum oil on percutaneous permeation of IM through excised rat skin Data are expressed as mean $\pm \operatorname{SD}(n=5)$
Based on the results of the penetration studies using two model drugs, it appears that $Z$. bungeanum oil could effectively facilitate the percutaneous absorptions of both hydrophilic and lipophilic drugs in a concentration-dependent manner, and also had a higher efficiency for the penetration of the hydrophilic drugs across the rat skin than for that of the lipophilic drugs according to the ER values. In addition, it is worth noting that the rat skin is more permeable than human skin due to the differences in their compositions and structure (Niazy, 1996), and more accurate evaluation should be measured using porcine skin or human skin (Reifenrath et al., 1984).

\subsection{Possible action mechanisms of $Z$. bungeanum oil}

The mechanisms of action of transdermal penetration enhancers have been investigated by many research groups and possible explanations for the enhancement activity have been suggested as follows: (1) alteration of the thermodynamic activity of the drug causing that drug molecules readily penetrate across the skin (Song et al., 2005; Zhang et al., 2006); (2) increased partition of the drug into the SC (Vaddi et al., 2002); (3) interactions with SC lipids or proteins resulting in disruption of the chemical structure or composition of the SC and disorganization of the highly ordered structures, thus increasing the delivery of the drug molecules (Williams and Barry, 2012). Certainly, other potential mechanisms for explaining the activity may also exist.

The results of the saturated solubilities and $\mathrm{SC} /$ vehicle partition coefficients of both 5-FU and IM after treatment with varying concentrations of $Z$. bungeanum oil are summarized in Tables 3,4 , and 5, respectively. For 5-FU, the saturated solubility slightly decreased with increasing the oil concentration, implying that the thermodynamic activity of 5-FU was altered to a certain extent in the presence of

Table 4 Percutaneous permeation parameters of IM through excised rat skin

\begin{tabular}{cccccc}
\hline Penetration enhancer & Flux $\left(\mu \mathrm{g} /\left(\mathrm{cm}^{2} \cdot \mathrm{h}\right)\right)$ & $T_{\mathrm{lag}}(\mathrm{h})$ & $Q_{24}\left(\mu \mathrm{g} / \mathrm{cm}^{2}\right)$ & ER & Solubility $(\mathrm{mg} / \mathrm{ml})$ \\
\hline Control & $0.40 \pm 0.03$ & $5.12 \pm 0.57$ & $16.52 \pm 1.71$ & 1.0 & $2.86 \pm 0.23$ \\
0.2 mol/L Azone & $16.36 \pm 2.49^{*}$ & $0.66 \pm 0.22^{*}$ & $708.84 \pm 107.34^{*}$ & 40.9 & $2.85 \pm 0.29$ \\
1\% oil & $5.84 \pm 0.68^{*}$ & $4.70 \pm 0.23$ & $215.74 \pm 33.58^{*}$ & 14.6 & $2.99 \pm 0.19$ \\
3\% oil & $21.80 \pm 3.77^{*}$ & $5.18 \pm 0.12$ & $837.24 \pm 71.72^{*}$ & 54.5 & $3.69 \pm 0.04^{*}$ \\
$5 \%$ oil & $28.78 \pm 3.54^{*}$ & $3.30 \pm 0.26^{*}$ & $978.42 \pm 87.42^{*}$ & 71.9 & $3.95 \pm 0.13^{*}$ \\
10\% oil & $33.06 \pm 4.27^{*}$ & $1.89 \pm 0.60^{*}$ & $1164.10 \pm 216.50^{*}$ & 82.6 & $4.43 \pm 0.22^{*}$ \\
\hline
\end{tabular}

Each value represents the mean $\pm \mathrm{SD}(n=5)$, except for ER. ${ }^{*} P<0.05$, statistically significant difference between enhancers and control 
Table 5 Partition coefficients of model drugs treated with different enhancers $(n=3)$

\begin{tabular}{ccclcc}
\hline Penetration & \multicolumn{2}{c}{5 -FU } & & \multicolumn{2}{c}{ IM } \\
\cline { 2 - 3 } \cline { 5 - 6 } enhancer & $K$ & PER & & $K$ & PER \\
\hline Control & $0.109 \pm 0.014$ & - & & $0.170 \pm 0.020$ & - \\
$1 \%$ oil & $0.101 \pm 0.008$ & 0.92 & & $0.202 \pm 0.061^{*}$ & 1.19 \\
$3 \%$ oil & $0.109 \pm 0.017$ & 0.99 & & $0.301 \pm 0.018^{*}$ & 1.77 \\
$5 \%$ oil & $0.110 \pm 0.007$ & 1.00 & & $0.477 \pm 0.147^{*}$ & 2.80 \\
$10 \%$ oil & $0.105 \pm 0.014$ & 0.96 & & $1.001 \pm 0.211^{*}$ & 5.86 \\
\hline
\end{tabular}

$K$ values are expressed as mean \pm SD $(n=3) .{ }^{*} P<0.05$, statistically significant difference between enhancers and control. $K$ : partition coefficient; PER: penetration enhancement ratio, and $\mathrm{PER}=$ (partition coefficient with essential oil treatment)/(partition coefficient without essential oil treatment)

the essential oil (Zhang et al., 2006). The saturated solubility of the 5-FU in the low-concentration oil $(1 \%$ and $3 \%)$ was higher than that of the control, which was probably due to its moderate hydrophilicity. However, there was no significant difference between the control and the $Z$. bungeanum oil at different concentrations in terms of the $\mathrm{SC} /$ vehicle partition coefficient, which indicates that $Z$. bungeanum oil might contribute negligibly to the partitioning of hydrophilic drugs into SC due to its lipophilicity properties. Contrary to 5-FU, the $\mathrm{SC} /$ vehicle partition coefficient of IM gradually increased with the oil concentration increasing, and it was up to 5.85 times higher than that of the control when the oil concentration reached $10 \%$. Furthermore, Z. bungeanum oil at different concentrations gradually improved the saturation solubility of IM, suggesting that the thermodynamic activity of IM was also significantly altered. Therefore, it implied that the $Z$. bungeanum oil could change the saturated solubility or the $\mathrm{SC} /$ vehicle partition coefficient of drugs to improve their delivery through the skin.

However, compared with the penetration ER, the alteration in the $\mathrm{SC} /$ vehicle partition coefficient or thermodynamic activity of two model drugs was relatively weak, suggesting that the $Z$. bungeanum oil probably enhanced the drug transdermal delivery substantially by changing the skin barrier property, but not altering the drug properties. Considering that $T_{\text {lag }}$ is related to diffusivity, and it can reflect changes in both the length of the permeation path $(h)$ and the diffusion coefficient $(D)$ to suggest the interaction of the enhancer with the SC lipid and protein according to Eq. (2) as described above. As shown in Tables 3 and 4 , for both of 5-FU and IM, the $T_{\text {lag }}$ values were roughly reduced with increasing the oil concentrations, indicating that the $Z$. bungeanum oil probably interacted with the SC lipids or protein to disrupt its barrier properties.

The FTIR absorption spectra were thus measured to investigate the biophysical changes of the rat skin SC properties due to its advantages on obtaining information about the lipids and keratin conformation in the SC. A representative spectrum of normal untreated rat skin $\mathrm{SC}$ shows that the major absorption peaks around 2850 and $2918 \mathrm{~cm}^{-1}$ due to symmetric and asymmetric $\mathrm{C}-\mathrm{H}$ stretching vibration of the lipid alkyl chains (Zhang et al., 2007), the fatty acid carbonyl stretching peaks near $1733 \mathrm{~cm}^{-1}$ (Moore and Rerek, 2000; Jain et al., 2002), and two strong amide absorption peaks in the range of $1500-1700 \mathrm{~cm}^{-1}$, i.e., $1652 \mathrm{~cm}^{-1}$ due to $\mathrm{C}=\mathrm{O}$ stretching vibration of the amide I band and $1538 \mathrm{~cm}^{-1}$ due to the $\mathrm{N}-\mathrm{H}$ bending vibration of the amide II band (Khurana et al., 2013). The shift to a higher frequency occurs when the $\mathrm{CH}_{2}$ groups along the alkyl chain of lipids change from trans to gauche conformation, suggesting that the SC lipid is disturbed, and the peak areas of the two C-H absorption bands are proportional to the amount of the SC lipids (Zhang et al., 2007). The change in the amide I and amide II absorption peaks to a lower wavelength is observed to indicate the alterations of the keratin conformation under the effect of penetration enhancers (Zbytovská et al., 2004; He et al., 2009). In addition, the frequencies of the fatty acid carbonyl stretching mode, the keratin amide I and amide II modes are sensitive to hydrogen bonding, which can provide information on head-group interactions in the SC lipids (Moore and Rerek, 2000).

The FTIR absorption spectra of rat SC treated with varying concentrations of the $Z$. bungeanum oil are displayed in Fig. 4. Compared with untreated rat SC, FTIR of rat SC treated with the solvent showed no obvious shift in wavenumber or changes in peak area or peak height, suggesting that PG:water (80:20, $\mathrm{v} / \mathrm{v})$ system was inefficient to perturb or extract the SC lipids and proteins. The decrease in peak area of the $\mathrm{C}-\mathrm{H}$ stretching bands and the shift in wavenumber were observed after treatment with different concentrations of $Z$. bungeanum oil, and the higher the oil concentration was, the greater the shift in $\mathrm{C}-\mathrm{H}$ stretching vibration was (as presented in Fig. 5 and Table 6). This indicated that the $Z$. bungeanum oil 


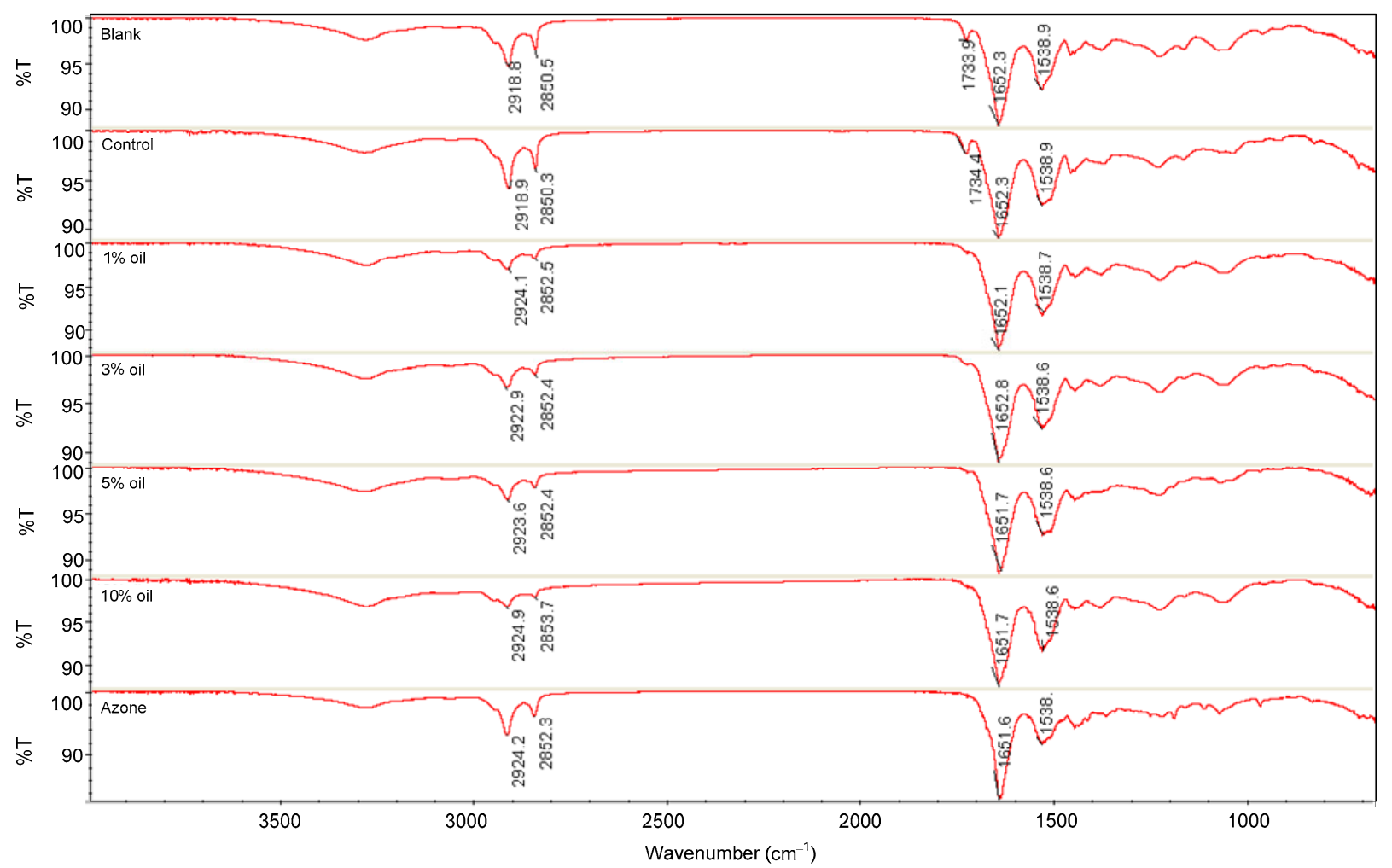

Fig. 4 FTIR spectra of the rat $\mathrm{SC}$ after treatment with different agents for $12 \mathrm{~h}$

Table 6 Peak positions of lipids and amides of SC after treated with varying agents

\begin{tabular}{ccccccc}
\hline \multirow{2}{*}{ Sample } & \multicolumn{2}{c}{$\begin{array}{c}\text { Peak position of } \\
\text { lipid }\left(\mathrm{cm}^{-1}\right)\end{array}$} & & \multicolumn{2}{c}{$\begin{array}{c}\text { Peak position of } \\
\text { amide }\left(\mathrm{cm}^{-1}\right)\end{array}$} \\
\cline { 2 - 3 } & $\begin{array}{c}\text { Asymmetric } \\
\text { C-H stretching }\end{array}$ & $\begin{array}{c}\text { Symmetric } \\
\text { Cy stretching }\end{array}$ & I & II \\
\hline Blank & 2918.87 & 2850.45 & & 1652.28 & 1538.98 \\
Control & 2918.92 & 2850.35 & & 1652.25 & 1538.93 \\
$1 \%$ oil & 2924.08 & 2852.57 & & 1652.06 & 1538.71 \\
$3 \%$ oil & 2922.99 & 2852.39 & & 1651.88 & 1538.60 \\
$5 \%$ oil & 2923.59 & 2852.38 & & 1651.79 & 1538.60 \\
$10 \%$ oil & 2924.97 & 2853.65 & & 1651.79 & 1538.56 \\
$\begin{array}{c}0.2 \text { mol/L } \\
\text { Azone }\end{array}$ & 2924.23 & 2853.59 & & 1651.70 & 1538.72 \\
\hline
\end{tabular}

perturbed and extracted the SC lipids to alter the skin permeability. It was notable that the presence of $0.2 \mathrm{~mol} / \mathrm{L}$ Azone only resulted in the shift of $\mathrm{C}-\mathrm{H}$ stretching vibration to high wavenumber, whereas the peak areas of the two $\mathrm{C}-\mathrm{H}$ stretching vibration absorption bands exhibited negligible change in comparison to those of untreated rat SC, which implied that $0.2 \mathrm{~mol} / \mathrm{L}$ Azone increased the drug permeation mainly by perturbing the SC lipids. Meanwhile, the absence of $-\mathrm{C}=\mathrm{O}$ peak of fatty acid indicated that the

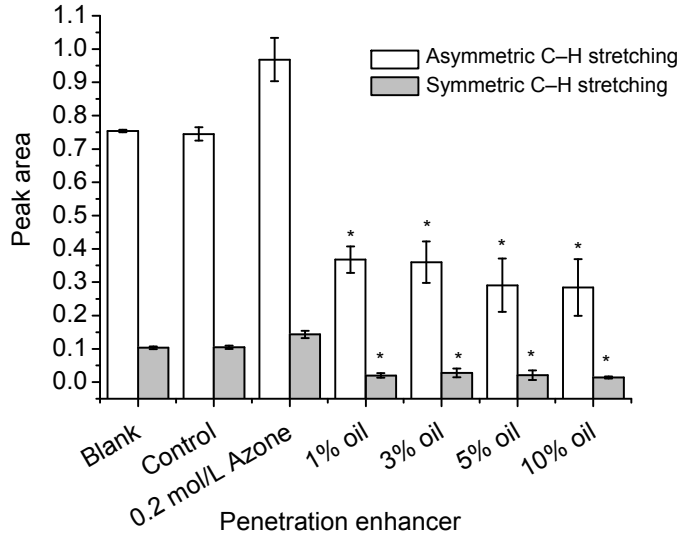

Fig. 5 Peak areas of $\mathrm{C}-\mathrm{H}$ stretching of SC lipids treated with different penetration enhancers

Data are expressed as mean $\pm \mathrm{SD}(n=3) .{ }^{*}$ Statistically significant difference between enhancers and control $(P<0.05)$

changes in the hydrogen bonding of the polar regions of the lipid bilayers might occur after treated with either Z. bungeanum oil or Azone. The wavelength movements of the amide I and amide II bands were narrow, perhaps indicating the $Z$. bungeanum oil contributed negligibly to the alteration of the keratin conformation. 


\section{Conclusions}

Based on the results of this study, it was found that $Z$. bungeanum oil appeared to be a low skin toxicity in comparison to the well-established and standard penetration enhancer Azone, and effectively facilitated the percutaneous permeation of both 5-FU and IM through rat skin in a concentration-dependent manner The partition studies exhibited that the partition coefficient for 5-FU was not altered, whereas it significantly increased for IM with incorporation of $Z$. bungeanum oil, indicating possible interaction of the oil with the SC lipids. Contrary to 5-FU, the saturated solubility of IM was gradually decreased with the increasing of the oil concentration, implying that the $Z$. bungeanum oil had altered the thermodynamic activities of both hydrophilic and lipophilic drugs to some extent. The results of FTIR studies showed that $Z$. bungeanum oil facilitated the drug permeation mainly by perturbing and extracting the SC lipids. In conclusion, $Z$. bungeanum oil could effectively facilitate the permeation of both hydrophilic and lipophilic drugs with low cytotoxicity.

\section{Acknowledgements}

The authors thank Ms. Li-xing YANG (Institute of Chinese Materia Medica, China Academy of Chinese Medical Sciences, Beijing, China) for her assist in the GC-MS analysis.

\section{Compliance with ethics guidelines}

Yi LAN, Qing WU, Ying-qiu MAO, Qiong WANG, Jing AN, Yan-yan CHEN, Wen-ping WANG, Bo-chen ZHAO, Na LIU, and Ye-wen ZHANG declare that they have no conflict of interest.

All institutional and national guidelines for the care and use of laboratory animals were followed.

\section{References}

Aqil, M., Ahad, A., Sultana, Y., et al., 2007. Status of terpenes as skin penetration enhancers. Drug Discov. Today, 12(23-24):1061-1067. [doi:10.1016/j.drudis.2007.09.001]

Batheja, P., Sheihet, L., Kohn, J., et al., 2011. Topical drug delivery by a polymeric nanosphere gel: formulation optimization and in vitro and in vivo skin distribution studies. J. Control. Release, 149(2):159-167. [doi:10.1016/j. jconrel.2010.10.005]

Fox, L.T., Gerber, M., Plessis, J.D., et al., 2011. Transdermal drug delivery enhancement by compounds of natural origin. Molecules, 16(12):10507-10540. [doi:10.3390/ molecules161210507]

Gong, Y.W., Huang, Y.F., Zhou, L.G., et al., 2009. Chemical composition and antifungal activity of the fruit oil of Zanthoxylum bungeanum Maxim. (Rutaceae) from China. J. Essent. Oil Res., 21(2):174-178. [doi:10.1080/1041 2905.2009.9700141]

He, W., Guo, X., Zhang, M., 2008. Transdermal permeation enhancement of $n$-trimethyl chitosan for testosterone. Int. J. Pharm., 356(1-2):82-87. [doi:10.1016/j.ijpharm.2007. 12.050]

He, W., Guo, X., Xiao, L., et al., 2009. Study on the mechanisms of chitosan and its derivatives used as transdermal penetration enhancers. Int. J. Pharm., 382(1-2):234-243. [doi:10.1016/j.ijpharm.2009.07.038]

Jain, A.K., Thomas, N.S., Panchagnula, R., 2002. Transdermal drug delivery of imipramine hydrochloride: I. Effect of terpenes. J. Control. Release, 79(1-3):93-101. [doi:10. 1016/S0168-3659(01)00524-7]

Kaushik, D., Costache, A., Michniak-Kohn, B., 2010. Percutaneous penetration modifiers and formulation effects. Int. J. Pharm., 386(1-2):42-51. [doi:10.1016/j.ijpharm. 2009. 10.052]

Khurana, S., Jain, N.K., Bedi, P.M.S., 2013. Nanoemulsion based gel for transdermal delivery of meloxicam: physicochemical, mechanistic investigation. Life Sci., 92(6-7): 383-392. [doi:10.1016/j.lfs.2013.01.005]

Magnusson, B.M., Runn, P., Koskinen, L.O., 1997. Terpeneenhanced transdermal permeation of water and ethanol in human epidermis. Acta Derm. Venereol., 77(4):264-267.

Meidan, V.M., Al-Khalili, M., Michniak, B.B., 2003. Enhanced iontophoretic delivery of buspirone hydrochloride across human skin using chemical enhancers. Int. J. Pharm., 264(1-2):73-83. [doi:10.1016/S0378-5173(03) 00390-9]

Moore, D.J., Rerek, M.E., 2000. Insights into the molecular organization of lipids in the skin barrier from infrared spectroscopy studies of stratum corneum lipid models. Acta Derm. Venereol., Suppl. (Stockh.), 208:16-22.

Narishetty, S.T.K., Panchagnula, R., 2005. Effect of L-menthol and 1,8-cineole on phase behavior and molecular organization of SC lipids and skin permeation of zidovudine. $J$. Control. Release, 102(1):59-70. [doi:10.1016/j.jconrel. 2004.09.016]

Niazy, E.M., 1996. Differences in penetration-enhancing effect of Azone through excised rabbit, rat, hairless mouse, guinea pig and human skins. Int. J. Pharm., 130(2):225-230. [doi:10.1016/0378-5173(95)04319-5]

Okabe, H., Takayama, K., Ogura, A., et al., 1989. Effect of limonene and related compounds on the percutaneous absorption of indomethacin. Drug Des. Deliv., 4(4):313321.

Reifenrath, W.G., Chellquist, E.M., Shipwash, E.A., et al., 1984. Evaluation of animal models for predicting skin penetration in man. Fundam. Appl. Toxicol., 4(2 Pt 2): S224-S230. [doi:10.1016/0272-0590(84)90156-8]

Sapra, B., Jain, S., Tiwary, A., 2008. Percutaneous permeation enhancement by terpenes: mechanistic view. AAPS J., 10(1):120-132. [doi:10.1208/s12248-008-9012-0] 
Sereshti, H., Izadmanesh, Y., Samadi, S., 2011. Optimized ultrasonic assisted extraction-dispersive liquid-liquid microextraction coupled with gas chromatography for determination of essential oil of Oliveria decumbens Vent J. Chromatogr. A, 1218(29):4593-4598. [doi:10.1016/j. chroma.2011.05.037]

Singh, J., Tripathi, K.P., Sakya, T.R., 1993. Effect of penetration enhancers on the in vitro transport of ephedrine through rat skin and human epidermis from matrix based transdermal formulations. Drug Dev. Ind. Pharm., 19(13): 1623-1628. [doi:10.3109/03639049309069331]

Song, Y., Xiao, C., Mendelsohn, R., et al., 2005. Investigation of iminosulfuranes as novel transdermal penetration enhancers: enhancement activity and cytotoxicity. Pharm. Res., 22(11):1918-1925. [doi:10.1007/s11095-005-7416-4]

Vaddi, H.K., Ho, P.C., Chan, Y.W., et al., 2002. Terpenes in ethanol: haloperidol permeation and partition through human skin and stratum corneum changes. J. Control. Release, 81(1-2):121-133. [doi:10.1016/S0168-3659(02) 00057-3]

Wang, Z., Ding, L., Li, T., et al., 2006. Improved solvent-free microwave extraction of essential oil from dried Cuminum cyminum L. and Zanthoxylum bungeanum Maxim. $J$. Chromatogr. A, 1102(1-2):11-17. [doi:10.1016/j.chroma. 2005.10.032]

Wei, S.G., Zhang, H.H., Wang, Y.Q., et al., 2011. Ultrasonic nebulization extraction-heating gas flow transfer-headspace single drop microextraction of essential oil from pericarp of Zanthoxylum bungeanum Maxim. J. Chromatogr. A, 1218(29):4599-4605. [doi:10.1016/j.chroma.2011.05.047]

Williams, A.C., Barry, B.W., 2012. Penetration enhancers. Adv. Drug Del. Rev., 64(Suppl.):128-137. [doi:10.1016/ j.addr.2012.09.032]
Wu, Q.F., Wang, W., Dai, X.Y., et al., 2012. Chemical compositions and anti-influenza activities of essential oils from Mosla dianthera. J. Ethnopharmacol., 139(2): 668-671. [doi:10.1016/j.jep.2011.11.056]

Xia, L., You, J., Li, G., et al., 2011. Compositional and antioxidant activity analysis of Zanthoxylum bungeanum seed oil obtained by supercritical $\mathrm{CO}_{2}$ fluid extraction. $J$. Am . Oil Chem. Soc., 88(1):23-32. [doi:10.1007/s11746-0101644-4]

Xiang, W., Gao, A., Liang, H., et al., 2010. Reversal of $P$-glycoprotein-mediated multidrug resistance in vitro by milbemycin compounds in adriamycin-resistant human breast carcinoma (MCF-7/adr) cells. Toxicol. in Vitro, 24(6):1474-1481. [doi:10.1016/j.tiv.2010.07.020]

Zbytovská, J., Raudenkolb, S., Wartewig, S., et al., 2004. Phase behaviour of transkarbam 12. Chem. Phys. Lipids, 129(1):97-109. [doi:10.1016/j.chemphyslip.2003.12.004]

Zhang, C.F., Yang, Z.L., Luo, J.B., et al., 2007. Effects of cinnamene enhancers on transdermal delivery of ligustrazine hydrochloride. Eur. J. Pharm. Biopharm., 67(2): 413-419. [doi:10.1016/j.ejpb.2007.02.019]

Zhang, L.C., Hu, J.H., Li, L., et al., 2006. In vivo and in vitro evaluation of essential oils from Ligusticum chuanxiong HORT on the transdermal delivery of flurbiprofen in rabbits. Biol. Pharm. Bull., 29(6):1217-1222. [doi:10. 1248/bpb.29.1217]

Zhao, L., Fang, L., Xu, Y., et al., 2008. Effect of $o$-acylmenthol on transdermal delivery of drugs with different lipophilicity. Int. J. Pharm., 352(1-2):92-103. [doi:10.1016/j.ijpharm.2007.10.017]

Zhu, R.X., Zhong, K., Zeng, W.C., et al., 2011. Essential oil composition and antibacterial activity of Zanthoxylum bungeanum. Afr. J. Microbiol. Res., 5(26):4631-4637. [doi:10.5897/AJMR11.772]

\section{中文概要:}

\section{本文题目：花椒挥发油作为天然经皮促透剂的细胞毒性及促透活性}

Cytotoxicity and enhancement activity of essential oil from Zanthoxylum bungeanum Maxim. as a natural transdermal penetration enhancer

研究目的：研究花椒挥发油作为天然经皮促透剂的促透活性及其促透机制, 同时评价其皮肤细胞毒性。

创新要点: 首次评价了花椒挥发油的皮肤细胞毒性、经皮促透活性及其作用机制。

研究方法: 利用表皮角质形成细胞 (HaCaT) 和真皮成纤维细胞 (CCC-ESF-1) 评价花椒挥发油的细胞 毒性; 采用亲水性及亲脂性模型药测定挥发油促透活性的基础上研究其促透作用机制。

重要结论: 花椒挥发油具有良好促透活性并具有较低皮肤细胞毒性。

关键词组: 花椒挥发油; 天然经皮促透剂; 透皮吸收; 表皮角质形成细胞（HaCaT）; 傅利叶变换红外 光谱 (ATR-FTIR) 